\title{
RESEARCH PROBLEMS
}

10. Solomon W. Golomb and Edward C. Posner: Hypercubes of non-negative integers.

It is known that if an $n \times n$ matrix of non-negative integers has the "rook-domain property" then the sum of its entries is at least $n^{2} / 2$ (see Trans. Inst. Electrical and Electronic Engineers, Information Theory, Vol. IT-10, 1964, p. 2051 for two proofs of this result of Joel N. Franklin and Alfred W. Hales). The rook-domain property means the following. The rook-domain of a point in a cartesian product of sets is the set of points all of whose coordinates but at most one agree with the coordinates of the given point. The rook-domain property asserts that the sum of all the entries in the rook-domain of every zero in a $k$-dimensional $n \times n \times \cdots \times n$ hypermatrix of non-negative integers is at least $n$ (obvious correspondence between hypermatrices and cartesian products defines "rook-domain" here).

Problem. Prove that if an $n^{k}$ hypermatrix of non-negative integers has the rook-domain property, then the sum of all its $n^{k}$ entries is at least $n^{k} / k$.

REMARKs. A solution would rule out the existence of certain combinatorial configurations. The result is true for $k=2$ allowing unequal sides (obvious modification in statement), and is presumably true in higher dimensions for unequal sides. There is a way of looking at the problem which makes it sort of a Fubini Theorem. (Received February $5,1965$.

\section{J. de Groot: Set theory.}

The number of open sets af a metrizable space is a power of two. Does the same hold for a Hausdorff space or for a compact Hausdorff space? (Received April 1, 1965.) 